\title{
Conjunctival melanoma after excision of a lentigo maligna melanoma in the ipsilateral eyelid skin
}

\author{
C Hicks, C Liu, M Hiranandani, A Garner, J Hungerford
}

\begin{abstract}
Moorfields Eye Hospital,
London

C Hicks

C Liu

$\mathrm{J}$ Hungerford
\end{abstract}

Herts and Essex General Hospital, Bishops

Stortford

M Hiranandani

Institute of

Ophthalmology, London

A Garner

Correspondence to:

Mr J Hungerford, Moorfields Eye Hospital, City Road, London ECIV 2PD.

Accepted for publication 26 August 1993

Figure 1 Pigmented lesion (lentigo maligna melanoma), left lower eyelid skin.

Figure 2 Pigmented lesion, bulbar conjunctiva (arrowed). Pigmentation is also present in the lower eyelid skin graft, spreading into adjacent lid skin.

Figure 3 Atypical acquired melanosis, shown by clusters of abnormal melanocytes in the epithelium, migrating away from the basement membrane. Squamous metaplasia of the epithelium, with loss of goblet cells, is also seen. Haematoxylin and eosin. Magnification $\times 300$.

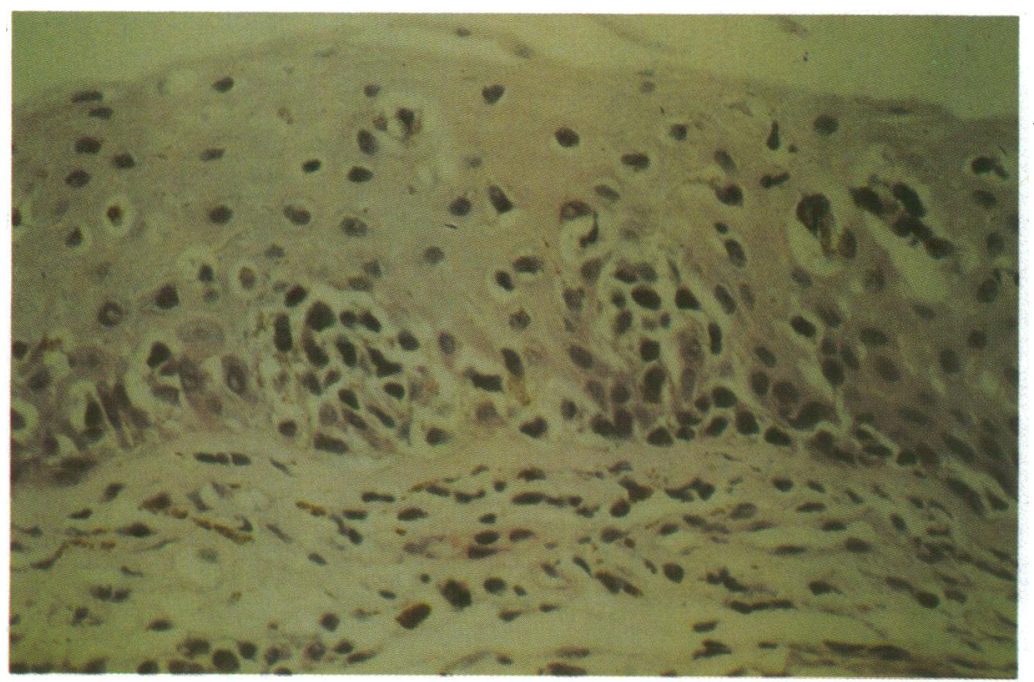

We present an unusual case in which conjunctival melanoma was preceded by excision of a lentigo maligna melanoma of the ipsilateral lower eyelid skin, and in which melanosis secondarily spread back into the grafted eyelid skin.

\section{Case report}

The patient was a white male who initially presented at the age of 60 , with a pigmented lesion on the left lower eyelid skin that had been present for 1 year (Fig 1). An excision biopsy was performed and the defect closed with a flap taken from the upper eyelid. Histological findings were of lentigo maligna melanoma to Clarke level II, with a maximum depth of $0.4 \mathrm{~mm}$, and atypia extending to the excision margin. The entire flap was excised and replaced with a Wolfe graft. Histology of this second specimen showed no residual tumour.

He was kept under review and referred to the oncology service at Moorfields Eye Hospital when, 3 years later, he developed a pigmented lesion on the interpalpebral bulbar conjunctiva of the left eye (Fig 2). Clinically this was diagnosed as primary acquired melanosis (PAM). There was an area within it of tethering, with increased thickness and vascularity, which was excised and the surrounding area treated with cryotherapy. It was also observed that pigmentation had recurred in the recipient lower eyelid skin, and spread contiguously into the Wolfe graft. There were, however, no thickened areas suggestive of a recurrence of the melanoma within the eyelid skin and no further skin biopsies were taken.

Histology of the conjunctival lesion revealed melanosis with atypia and microscopic invasive melanoma (Fig 3). When stained for S-100 protein (Fig 4), which identifies cells of neural crest origin, labelled cells were seen to invade the superficial stroma. PAM with atypia was seen to extend to the surgical margin.

Within 1 year further suspicious areas developed within the acquired melanosis on the bulbar conjunctiva and were excised. These revealed an area of PAM with atypia in the upper fornix (Fig 5) and an early malignant melanoma arising from PAM in the lower fornix (Fig 6). The areas surrounding the biopsy sites were treated with further cryotherapy.

In spite of the spreading pigmentation within the eyelid skin, there has been no evidence of a recurrence of the skin melanoma. It is hoped that regular examination under anaesthesia, allowing double eversion and, if necessary, excision of any suspicious conjunctival lesions and cryotherapy to any surrounding diffuse areas of PAM, will contain this patient's disease, thereby preventing or deferring entry into an aggressive stage which would require mutilating surgery. ${ }^{1-4}$ 


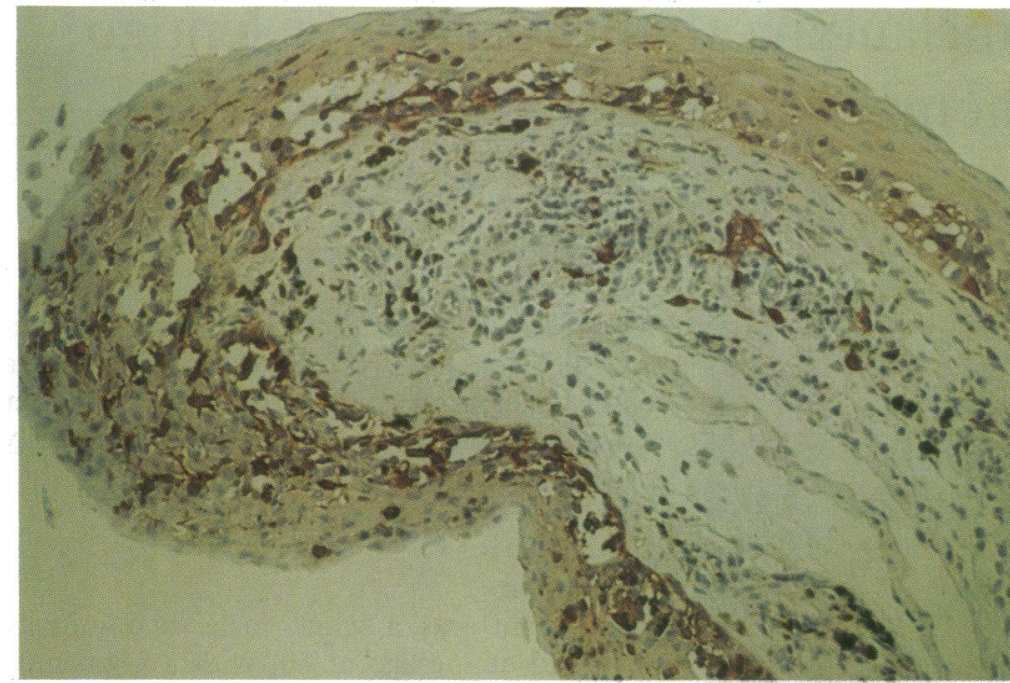

Figure 4 Specimen stained for S-100 protein, showing labelled cells invading the superficial stroma. Immunoperoxidase. Magnification $\times 120$.

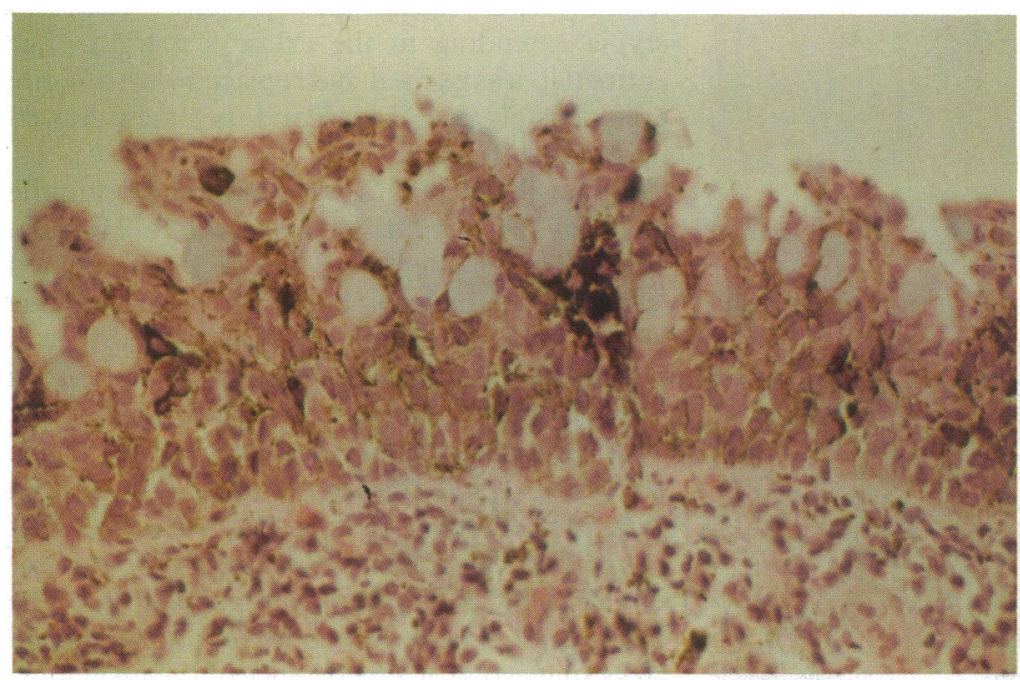

Figure 5 Primary acquired melanosis with atypia, showing melanocytes migrating away from the basement membrane. Goblet cells and inflammatory cells are also conspicuous. Haematoxylin and eosin. Magnification $\times 300$.

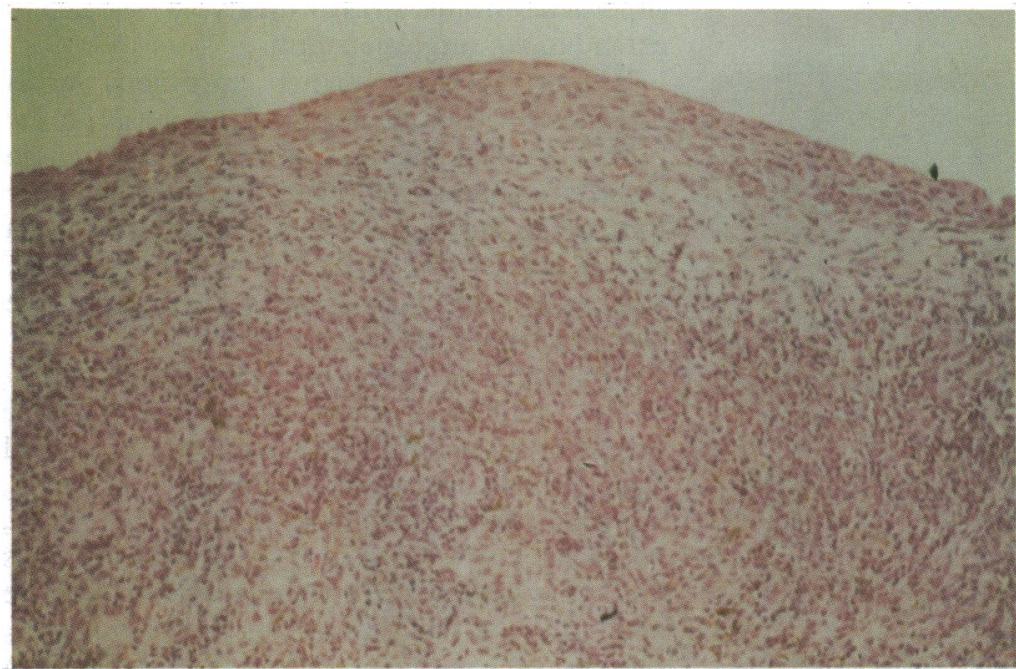

Figure 6 Early malignant melanoma, showing stromal invasion by melanocytes. Haematoxylin and eosin. Magnification $\times 30$.

\section{Comment}

Hutchinson, who first described the 'senile freckle' in 1892,5 noted that the eyelid was a common site, and that the enlarging lesion may extend into the adjacent conjunctiva. Such spread appears, however, to be unusual and more commonly spread occurs from conjunctiva to lid. Giblin $e^{2}$ al ${ }^{6}$ reported two cases in which cutaneous eyelid melanoma preceded conjunctival malignant melanoma. They suggest that the two apparently separate entities may have derived originally from inapparent patches of the rarer non-pigmented variant of PAM, primary acquired melanosis sine pigmento. The abnormal cells, it is suggested, may have originated within the conjunctiva and migrated into the cutaneous epithelium, only later developing pigment in both sites.

Certainly the mobility of melanoma cells is well established and this property, along with the possibility of extensive PAM sine pigmento surrounding PAM, may explain the frequency with which PAM recurs after local treatment. Alternatively, the clinical progression in this case might be explained by the multifocal nature of PAM, which can be regarded as a generalised defect in melanocyte function. If that is the case, it is interesting that the disease is unilateral and perhaps surprising that the association of an eyelid, with a separate conjunctival melanoma, is not more common.

This case underlines the importance of examination under anaesthesia, with particularly careful inspection of the conjunctival fornices, in all patients with an eyelid melanoma or conjunctival PAM.

1 Jakobiec FA, Brownstein S, Wilkinson RD, Khalil M, Cooper WC, Shibata HR. Combined surgery and cryotherapy for diffuse mata HR. Combined surgery and cryotherapy for diffuse malignant melanom

2 Jakobiec FA. Conjunctival melanoma: unfinished busines [Editorial]. Arch Ophthalmol 1980; 98: 1378-484.

3 Jakobiec FA, Rini FJ, Fraunfelder FT, Brownstein S Cryotherapy for conjunctival primary acquired melanosis an malignant melanoma. Experience with 62 cases. Ophthamology 1988; 95: 1058-70.

4 Liesegang TJ, Campbell RJ. Mayo Clinic experience with conjunctival melanomas. Arch Ophthalmol 1980; 98: 1385-9.

5 Hutchinson J. Senile freckles. Arch Surg 1892; 3: 319.

6 Giblin ME, Shields CL, Shields JA, Eagle RC. Primary eyelid melanoma associated with primary conjunctival malignant melanoma. Aust NZ F Ophthalmol 1988; 16: 127-31. 